\title{
RSK2 signaling in brain habenula contributes to place aversion learning
}

\author{
Emmanuel Darcq, ${ }^{1}$ Pascale Koebel, Carolina Del Boca, Solange Pannetier, Anne-Sophie \\ Kirstetter, Jean-Marie Garnier, André Hanauer, Katia Befort, and Brigitte L. Kieffer ${ }^{2}$ \\ Institut de Génétique et de Biologie Moléculaire et Cellulaire, Centre National de la Recherche Scientifique/Institut National de la Santé \\ et de la Recherche Médicale/Université de Strasbourg, 67404 Illkirch, France
}

\begin{abstract}
RSK2 is a Ser/Thr kinase acting in the Ras/MAPK pathway. Rsk2 gene deficiency leads to the Coffin-Lowry Syndrome, notably characterized by cognitive deficits. We found that mrsk2 knockout mice are unable to associate an aversive stimulus with context in a lithium-induced conditioned place aversion task requiring both high-order cognition and emotional processing. Virally mediated shRNA-RSK2 knockdown in the habenula, whose involvement in cognition is receiving increasing attention, also ablated contextual conditioning. RSK2 signaling in the habenula, therefore, is essential for this task. Our study reveals a novel role for RSK2 in cognitive processes and uncovers the critical implication of an intriguing brain structure in place aversion learning.
\end{abstract}

[Supplemental material is available for this article.]

The 90-kDa ribosomal S6 kinases (RSKs) constitute a family of four homologous Ser/Thr kinases (RSK1-4) that are activated by extracellular-regulated kinases 1 and $2(\mathrm{ERK} 1 / 2)$ in response to growth factors, hormones, chemokines, and neurotransmitters. RSKs, in turn, phosphorylate several cytosolic and nuclear targets and regulate a variety of cellular processes implicated in cell survival, growth, proliferation, and migration (Anjum and Blenis 2008). Many aspects of RSK function have been clarified at the level of cellular signaling, but the implications of RSK signaling in vivo remain largely unknown.

The important physiological role of RSK2 was uncovered by the discovery of rsk2 gene defects in the Coffin-Lowry Syndrome (CLS) (Trivier et al. 1996). This X-linked disorder is characterized by psychomotor retardation, digit and facial dymorphisms, and progressive skeletal deformations (Hanauer and Young 2002; Pereira et al. 2010). CLS is highly heterogeneous, and mental retardation is variable, but most male patients show severe cognitive impairment. Knockout mice for $r s k 2$ gene (mrsk2 KO) show some of the common features of CLS, and altered glycogen metabolism in skeletal muscle (Dufresne et al. 2001) or lipodystrophy (El-Haschimi et al. 2003) have been reported. At the behavioral level, mrsk $2 \mathrm{KO}$ mice show normal motor function and emotional responses but display deficits in working and long-term spatial memory, as well as learning impairments in exploratory behavior (Poirier et al. 2007). Together, therefore, both the human syndrome and the mouse phenotype reveal a role for RSK2 in learning and memory processes, in accordance with the reported brain distribution of this kinase in neocortex and hippocampus that are essential for cognitive function (Zeniou et al. 2002).

Here we investigated the role of RSK2 in the ability to associate a specific context with an aversive stimulus. This cognitive task involves place aversion learning, which is essential for species

\footnotetext{
1Present address: The Ernest Gallo Clinic and Research Center, University of California, San Francisco, Emeryville, CA 94608, USA. ${ }^{2}$ Corresponding author.

E-mail briki@igbmc.fr.

Article is online at http://www.learnmem.org/cgi/doi/10.1101/Im.2221011.
}

survival (Silva et al. 1998). We submitted mrsk2 KO mice (Yang et al. 2004) and their wild-type (WT) counterparts to a lithium-induced conditioned place aversion paradigm (CPA), as described previously (Le Merrer et al. 2011). Mutant mice and WT controls were conditioned to receive either saline or lithium (3 mEq/ $\mathrm{kg}$ ) and tested for CPA (Fig. 1). Three-way ANOVA showed a main effect of treatment $\left(F_{(1,56)}=9.27, P=0.004\right)$ but no effect of genotype $\left(F_{(1,56)}=1.74, P=0.193\right)$. There were significant genotype $\times$ treatment $\left(F_{(1,56)}=4.672, P=0.035\right)$, session $\times$ treatment $\left(F_{(1,56)}=11.76, P=0.001\right)$, and session $\times$ genotype $\times$ treatment $\left(F_{(1,56)}=5.131, \quad P=0.027\right) \quad$ interactions. Post-hoc comparisons of the post-conditioning session revealed no effect of genotype following saline; however, the mutant mice had significantly decreased CPA compared to the WT group following 3 $\mathrm{mEq} / \mathrm{kg} \mathrm{LiCl}$. These results show that lithium CPA is impaired in mrsk2 KO mice, indicating that the ability to form the drug/ context association is altered in these animals. RSK2 signaling, therefore, contributes to place aversion.

RSK2 is expressed during embryogenesis (Kohn et al. 2003; Dugani et al. 2010) and postnatally. In the adult, the strongest expression of RSK2 was reported in skeletal muscle, heart, and pancreas. In the brain, RSK2 was found at the level of the neocortex, hippocampus, and purkinge cells of the cerebellum in both mouse and human (Zeniou et al. 2002). To fine map RSK2 expression throughout the adult mouse brain, we hybridized a digoxigenin-labeled mrsk2 RNA probe to coronal brain sections from WT mice, and our data confirm expression of the mrsk 2 transcript in the cortex and throughout the hippocampus (Fig. 2A). Interestingly, we also observed substantial mrsk2 expression in the habenula (Hb) (Fig. 2A; Lecourtier and Kelly 2007; Hikosaka 2010). Labeling was prominent in the medial subdivision of $\mathrm{Hb}(\mathrm{MHb})$, indicating a transcript distribution restricted to $\mathrm{Hb}$ neurons receiving inputs from the limbic system and sending outputs to the interpeduncular nucleus projecting to the raphe (Hikosaka 2010). We further confirmed expression of RSK2 protein in the Hb complex by Western blot analysis (Fig. 2B). The 90-KDa immunolabelled protein was detectable at similar levels in microdissected cortex and $\mathrm{Hb}$ samples, and staining was absent in 


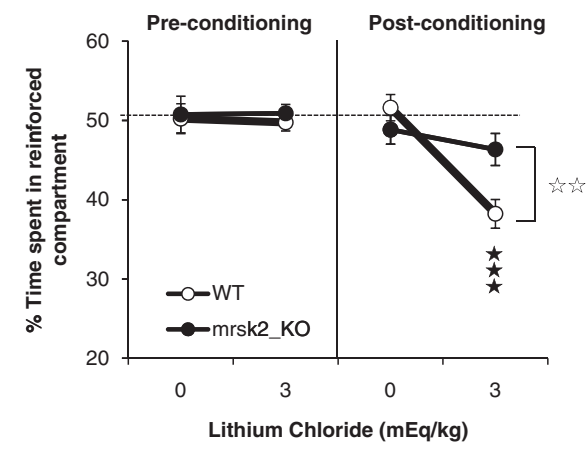

Figure 1. Lithium conditioned place aversion is impaired in mice lacking RSK2 gene (mrsk2 KO). Wild-type (WT) but not mrsk2 KO animals exhibit a significant place aversion for the compartment associated with $\mathrm{LiCl}$ when tested drug-free (WT: $n=16$ per dose; and KO: $n=14$ per dose). Data show mean ( \pm SEM) time spent in the drug-paired compartment (expressed as a percentage of time spent in the two compartments) during the 20-min pre- and post-conditioning sessions. Data were computed with the Statistical Program for the Social Sciences (SPSS; V.18.0) and analyzed using three-way analysis of variance (ANOVA), with genotype/shRNA and treatment as between-groups factors and session (pre- vs. post-conditioning) as a within-group factor. (See Supplemental Material for details.) (**) $P<0.05$, comparison between genotype; $(* *) P<0.001$, comparison to saline control.

samples from mrsk2 KO mice. Expression of RSK2 in the Hb has not been reported earlier. RSK2 may, therefore, contribute to cognitive processing via its broad expression in cortical and hippocampal neural network, and also through the modulation of $\mathrm{MHb}$ neuronal activity.

We then examined whether RSK2 signaling in Hb contributes to place conditioning, using virally mediated RSK2 shRNA knockdown. We first designed three shRNA sequences targeting distinct regions of mrsk2 mRNA (shRSK2-105, shRSK2-1112, and shRSK2-1954) and a shRNA containing scrambled sequence with no similarity with any known gene (shScramble) (see Supplemental Material for details). We compared in vitro efficacies of the three shRNAs by cotransfecting COS- 1 cells with two plasmids, one overexpressing the RSK2 protein in fusion with the fluorescent reporter dsRed (RSK2-dsRed) and the other expressing the shRNA together with a fluorescent eGFP reporter (see Supplemental Materials for details). One irrelevant shRNA was also tested. Following $2 \mathrm{~d}$ post-transfection, we evaluated RSK2 expression levels either by fluorescence-activated cell sorting (FACS) or Western blot analysis. FACS analysis of the red fluorescent signal showed reduced RSK2-dsRed expression in cells expressing each of the three shRNAs, compared to cells expressing either the scramble, irrelevant, or no shRNA (Fig. 2C). Consistent with this observation, Western blot analysis showed lower RSK2 immunoreactivity in the three mrsk2 shRNA-expressing samples compared to controls (Fig. 2C). In both cases, shRSK2-105 showed the best silencing activity and was, therefore, selected for insertion into the AAV2 viral vector using the AAV2 Helper-Free system (Stratagene) with some modifications (see Supplemental Material for details).

Next, we tested the AAV2-shRSK2 virus for in vivo RSK2 knockdown in $\mathrm{Hb}$. We injected viral particles stereotaxically into the Hb complex, as medially as possible (see Supplemental Materials for details). To directly compare the effects of the AAV2-shRSK2 with those of a control scrambled shRNA, several animals were injected bilaterally using AAV2-shRSK2 on one side and AAV2-shScramble on the other side. Three weeks following surgery, brains were removed and sections analyzed for eGFP fluorescence to detect virus spreading, mrsk2 mRNA expression, and cellular integrity. A representative section is shown in Figure 2D. For all animals $(n=4)$, fluorescence imaging showed strong expression of viral eGFP and intact DAPI staining on the two sides, confirming efficient viral infection and lack of histological damage following both AAV2-shRSK2 and AAV2-shScramble injections. Importantly, the mrsk2 transcript was clearly detectable on the control side, while labeling was barely detectable on the AAV2-shRSK2 side. These observations indicate that the stereotaxic injection procedure leads to efficient, accurate, and histologically innocuous viral infection in $\mathrm{MHb}$ and that the AAV2-shRSK2 potently reduces mrsk2 mRNA expression at the injection site. Separate animals were treated bilaterally to measure RSK2 knockdown at the protein level. The Hb was microdissected, and protein content was analyzed by Western Blot and quantified (Supplemental Fig. S1). EGFP staining was comparable for both AAV2-shRSK2 and AAV2- shScramble control samples, indicating efficient viral infection at the targeted brain site. RSK2 immunostaining was lower in AAV2-shRSK2 samples compared to AAV2shScramble samples (decrease of $39.48 \pm 9.04 \%, P=0.01, n=$ $8-9)$. Altogether, our results indicate that both transcript and protein levels are reduced following AAV2-shRSK2 injection.

These experimental conditions were, therefore, used to generate cohorts of virally infected animals for behavioral analysis. Two independent cohorts of 32 animals were prepared accordingly. For each cohort, animals were injected bilaterally either with AAV2-shRSK2 $(n=16)$ or with AAV2-shScramble $(n=16)$. After $6 \mathrm{wk}$, each cohort was subjected to lithium-induced conditioned place aversion, as previously done for RSK2 knockout mice ( $n=8$ per group and per drug). After behavioral testing, brains were removed and analyzed for correct injection site (Fig. 3A) and viral expression (eGFP signal) (Fig. 3B). Most animals showed intense eGFP expression in MHb. Few animals showed absent (two animals) or misplaced (four animals) eGFP signal at one side and were excluded from the behavioral statistical analysis. In situ hybridization analysis was performed on three animals from each group to confirm mrsk2 down-regulation (Fig. 3B).

Behavioral lithium CPA data from the two independent cohorts were pooled (Fig. 3C). A three-way ANOVA showed significant main effects of treatment $\left(F_{(1,54)}=12.52, P=0.001\right)$ and genotype $\left(F_{(1,54)}=4.33, P=0.042\right)$. There were significant session $\times$ treatment $\left(F_{(1,54)}=8.53, P=0.05\right)$ and session $\times$ genotype $\left(F_{(1,54)}=9.41, P=0.003\right)$ interactions. Post-hoc comparisons of the post-conditioning session revealed no effect of shRNA following saline; however, the AAV2-shRSK2-injected mice had a significant decrease in CPA compared to AAV2-shScramble-treated animals following $3 \mathrm{mEq} / \mathrm{kg} \mathrm{LiCl}$. Notably, global activity was not modified in AAV2-shRSK2-injected animals compared with their controls (see Supplemental Fig. S2), indicating that the stereotaxic procedure did not modify general exploratory activity that may interfere with the learning performance. Altogether, these results demonstrate that full expression of RSK2 in MHb is necessary for the place aversion response.

In conclusion, our study demonstrates that RSK2 signaling in $\mathrm{MHb}$ is essential for the formation of drug/context associations, and this observation has two important implications. First, the data reveal a novel role of RSK2 signaling in cognitive processes, and second, our findings uncover the critical implication of an intriguing brain structure in conditioned place aversion.

RSK2 is a serine/threonine kinase operating in the Ras/MAPK signaling pathway, which plays an important role in synaptic plasticity and memory (Davis and Laroche 2006). Mutations in the $r s k 2$ gene lead to the Coffin-Lowry Syndrome (Pereira et al. 2010), a severe X-linked disorder involving developmental, metabolic, and cognitive alterations, and rsk2 gene $\mathrm{KO}$ in the mouse recapitulates some aspects of the syndrome at both peripheral (Dufresne et al. 2001; El-Haschimi et al. 2003; Yang et al. 2004) and central levels (Poirier et al. 2007). Poirier and colleagues examined general behavior and cognitive processes in mrsk2 $\mathrm{KO}$ 
A
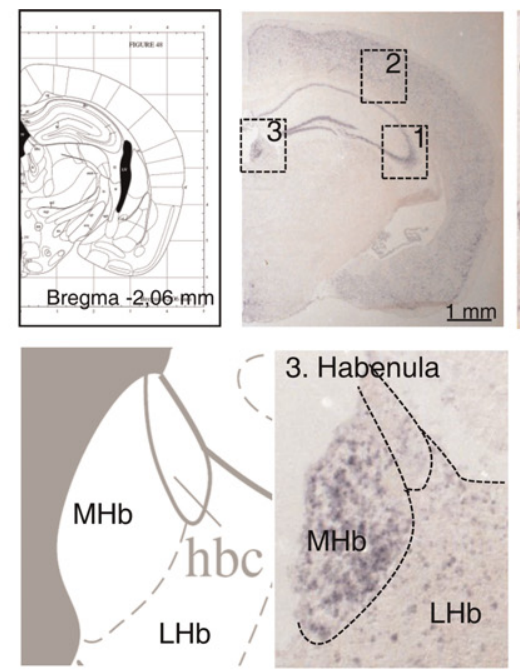

C
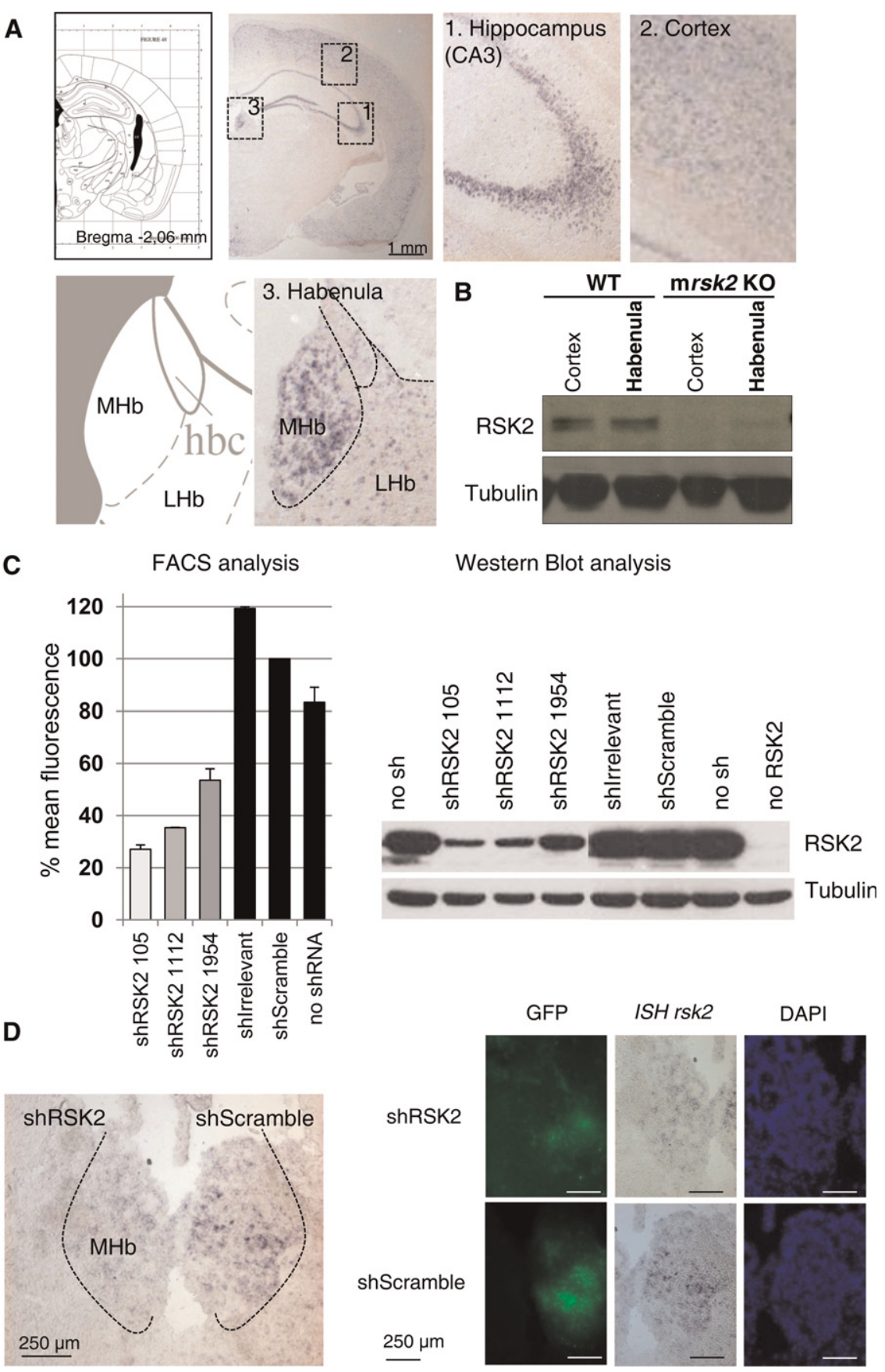

Western Blot analysis

Figure 2. Expression of RSK2 in medial habenula and AAV2-shRNA RSK2 validation. (A) In situ hybridization shows mrsk2 mRNA expression in hippocampus (1), cortex (2), and habenula (3). (B) RSK2 protein is expressed in cortex and habenula of WT mice but not KO mice, as revealed by Western blotting on microdissected preparations. (C) In vitro validation of AAV2-shRNA RSK2 knockdown, with expression level of RSK2-dsRed in COS-1 cells coexpressing mrsk2-targeted or control shRNAs, as measured by fluorescence-activated cell sorting (left) and Western blotting (right). shRSK2-105 shows the best silencing activity. (D) In vivo validation of AAV2-shRNA RSK2. AAV2-shScramble (right) or AAV2-shRSK2 (left) were injected stereotaxically in $\mathrm{Hb}(n=4)$. After $3 \mathrm{wk}$, brain sections were processed for detection of Green fluorescent protein (GFP) (left), mrsk2 mRNA (middle), and DAPI staining (right). AAV2-shRSK2, but not AAV2-shScramble, reduces mrsk2 mRNA expression in $\mathrm{Hb}$. A representative image is shown.

mice through two extensive test batteries. A main finding of their study was the performance impairment of mutant mice in the acquisition of several place navigation tasks, using the watermaze. A slight modification was also observed in the 8 -arm radial maze, and these two phenotypes indicate a role of RSK2 in spatial learning and working memory (Poirier et al. 2007). Mutant mice otherwise behaved normally in many other cognitive tasks (habituation of exploratory activity, hole-board task, and object exploration), suggesting that RSK2 modulates specific aspects of spatial learning. Behavioral data from Poirier and colleagues are consistent with the well-described RSK2 expression in hippocampus, a brain structure strongly implicated in spatial tasks. In this study, we examined mrsk2 $\mathrm{KO}$ mice in a different type of cognitive paradigm. Lithium place conditioning is a form of learning that requires both high-order cognitive integration of contextual cues and emotional processing of an aversive stimulus (Silva et al. 1998), which together contribute to the setting of drug/context association. The lack of lithium CPA in mrsk2 $\mathrm{KO}$ mice first demonstrates the importance of RSK2 in integrating contextual elements, consistent with hippocampal RSK2 expression and the previously reported role of RSK2 in spatial learning. In addition, this finding suggests a role for RSK2 in processing value-based information, and we propose that RSK2 signaling also regulates emotional aspects of learning and memory.

RSK2-mediated emotional learning may operate at nonhippocampal brain sites. Contextual conditioning recruits cortical, hippocampal, and amygdalar neural networks (Tzschentke 1998). Another less-well- known and intriguing brain structure possibly involved in contextual conditioning is the habenular complex. Lesion studies, electrophysiology, and imaging studies in humans converge to show a role for the $\mathrm{Hb}$ in place learning (Lecourtier et al. 2004), memory, and attention, as well as behavioral flexibility (Lecourtier and Kelly 2007). The Hb is a highly conserved brain structure and represents a key reciprocal node linking limbic and extrapyramidal systems (Lecourtier and Kelly 2007). It receives strong input from the basal ganglia (via the entopeduncular nucleus) and regulates ascending monomaminergic and cholinergic transmission throughout the forebrain. As such, the $\mathrm{Hb}$ is not only involved in cognitive behaviors but also in their motoric, motivational, and rewarding aspects and is essential in value-based decision-making (Hikosaka 2010).

Our observation of discrete and strong RSK2 expression in $\mathrm{MHb}$, in addition to other brain structures involved in cognitive tasks, led us to investigate whether habenular RSK2 could be involved in contextual conditioning. Our data demonstrate that RSK2 signaling in $\mathrm{MHb}$, indeed, is 
A

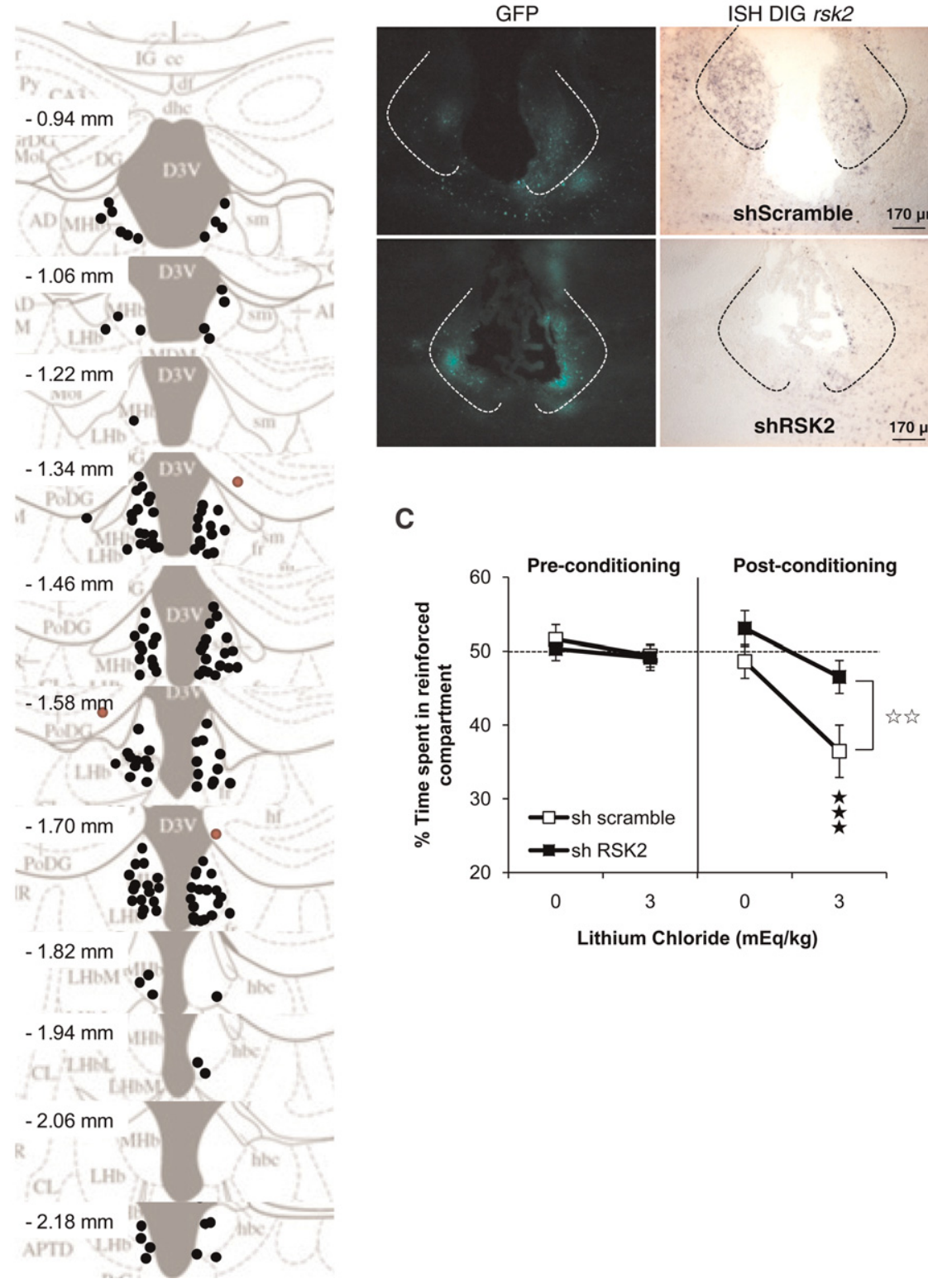

Figure 3. RSK2 knockdown in $\mathrm{Hb}$ decreases lithium conditioned place aversion. (A) Injection sites in the $\mathrm{Hb}$ (dark circle) and outside (red circle) were determined for all mice. Mice with injections visibly outside of $\mathrm{MHb}$ were excluded from statistical analysis. (B) Representative images showing viral expression (GFP) and intact (AAV2-shScramble) or reduced (AAV2-shRSK2) mrsk2 expression (analysis done for $n=3$ per group/each cohort). (C) LiCl CPA in mice injected with AAV2-shRSK2 in habenula (shRSK2) and their controls (shScramble). AAV2-shRSK2-injected mice failed to display lithium CPA. Data show mean $( \pm$ SEM) time spent in the drug-paired compartment (expressed as a percentage of time spent in the two compartments) during the 20-min pre- and post-conditioning sessions (AAV2-shRSK2, saline, $n=15$, lithium, $n=14$; AAV2-shScramble, saline, $n=14$, lithium, $n=15$ ). $\left(^{* *}\right) P<0.05$, comparison between shRNA; $\left(^{* * *}\right) P<0.001$, comparison to saline control.
There has been a surge of interest in the $\mathrm{Hb}$ network in the last several years; however, molecular events operating at the level of habenular neurons are largely unknown. The few existing studies have mainly investigated cholinergic transmission, since $\mathrm{Hb}$ nuclei ( $\mathrm{LHb}$ and $\mathrm{MHb}$ ) express high levels of nicotinic receptors, including $\alpha 2, \alpha 5$, and $\beta 4$ subunits. Pharmacological blockage of nicotinic receptors by mecamylamine decreases memory performance in a 16-arm maze upon injection in the LHb (Sanders et al. 2010) and induces nicotine withdrawal after antagonist administration targeting the MHbinterpeduncular system (Salas et al. 2009). Our study identifies RSK2 as a novel important molecular actor of $\mathrm{MHb}$, which regulates neural activity of the $\mathrm{Hb}$ complex in contextual conditioning. Upstream RSK2 activators, including cell surface membrane receptors and associated extracellularregulated kinases, and downstream effectors operating in $\mathrm{MHb}$ neurons remain to be clarified. Molecular genetics using simpler model organisms (Agetsuma et al. 2010) will further help in expanding our knowledge of molecular mechanisms underlying $\mathrm{Hb}$ function in the brain.

\section{Acknowledgments}

We wish to thank J. Le Merrer and A. Pradhan for behavioral advice and M. Mahoney and C. GavériauxRuff for careful reading of the manuscript. This work was supported by the Centre National de la Recherche Scientifique, Institut National de la Santé et de la Recherche Médicale, and Université de Strasbourg. We thank the European Union (GENADDICT/FP6 005166) and the National Institutes of Health (NIAAA AA-16658 and NIDA DA-16768) for financial support.

\section{References}

Agetsuma M, Aizawa H, Aoki T, Nakayama R,

Takahoko M, Goto M, Sassa T, Amo R,

Shiraki T, Kawakami K, et al. 2010. The

habenula is crucial for experience-

dependent modification of fear

responses in zebrafish. Nat Neurosci 13: 1354-1356.

necessary for this form of learning. Whether RSK2 activity in MHb is sufficient for this remains to be established. At this stage, we cannot exclude that RSK2 acting at other brain sites also contributes to contextual learning, and future studies will determine whether hippocampal or cortical RSK2 signaling is also involved in lithium CPA. Importantly here, our findings showing that both mrsk2 KO mice and AAV2-shRSK2-treated mice lack lithium $\mathrm{CPA}$ demonstrates for the first time that $\mathrm{MHb}$ is a critical neural substrate for place aversion. cellular signalling. Nat Rev Mol Cell Biol 9: 747-758.

Davis S, Laroche S. 2006. Mitogen-activated protein kinase/extracellular regulated kinase signalling and memory stabilization: A review. Genes Brain Behav 5 Suppl 2: 61-72.

Dufresne SD, Bjorbaek C, El-Haschimi K, Zhao Y, Aschenbach WG, Moller DE, Goodyear LJ. 2001. Altered extracellular signal-regulated kinase signaling and glycogen metabolism in skeletal muscle from p90 ribosomal S6 kinase 2 knockout mice. Mol Cell Biol 21: 81-87.

Dugani CB, Paquin A, Kaplan DR, Miller FD. 2010. Coffin-Lowry syndrome: A role for RSK2 in mammalian neurogenesis. Dev Biol 347: 348-359.
Anjum R, Blenis J. 2008. The RSK family of kinases: Emerging roles in 
El-Haschimi K, Dufresne SD, Hirshman MF, Flier JS, Goodyear LJ, Bjorbaek C. 2003. Insulin resistance and lipodystrophy in mice lacking ribosomal S6 kinase 2. Diabetes 52: 1340-1346.

Hanauer A, Young ID. 2002. Coffin-Lowry syndrome: Clinical and molecular features. J Med Genet 39: 705-713.

Hikosaka O. 2010. The habenula: From stress evasion to value-based decision-making. Nat Rev Neurosci 11: 503-513.

Kohn M, Hameister H, Vogel M, Kehrer-Sawatzki H. 2003. Expression pattern of the Rsk2, Rsk4, and $P d k 1$ genes during murine embryogenesis. Gene Expr Patterns 3: 173-177.

Le Merrer J, Plaza-Zabala A, Del Boca C, Matifas A, Maldonado R, Kieffer BL. 2011. Deletion of the delta opioid receptor gene impairs place conditioning but preserves morphine reinforcement. Biol Psychiatry 69: 700-703.

Lecourtier L, Kelly PH. 2007. A conductor hidden in the orchestra? Role of the habenular complex in monoamine transmission and cognition. Neurosci Biobehav Rev 31: 658-672.

Lecourtier L, Neijt HC, Kelly PH. 2004. Habenula lesions cause impaired cognitive performance in rats: Implications for schizophrenia. Eur J Neurosci 19: 2551-2560.

Pereira PM, Schneider A, Pannetier S, Heron D, Hanauer A. 2010. Coffin-Lowry syndrome. Eur J Hum Genet 18: 627-633.

Poirier R, Jacquot S, Vaillend C, Soutthiphong AA, Libbey M, Davis S, Laroche S, Hanauer A, Welzl H, Lipp HP, et al. 2007. Deletion of the Coffin-Lowry syndrome gene Rsk2 in mice is associated with impaired spatial learning and reduced control of exploratory behavior. Behav Genet 37: 31-50.
Salas R, Sturm R, Boulter J, De Biasi M. 2009. Nicotinic receptors in the habenulo-interpeduncular system are necessary for nicotine withdrawal in mice. J Neurosci 29: 3014-3018.

Sanders D, Simkiss D, Braddy D, Baccus S, Morton T, Cannady R, Weaver N, Rose JE, Levin ED. 2010. Nicotinic receptors in the habenula: Importance for memory. Neuroscience 166: 386-390.

Silva AJ, Giese KP, Fedorov NB, Frankland PW, Kogan JH. 1998. Molecular, cellular, and neuroanatomical substrates of place learning. Neurobiol Learn Mem 70: 44-61.

Trivier E, De Cesare D, Jacquot S, Pannetier S, Zackai E, Young I, Mandel JL, Sassone-Corsi P, Hanauer A. 1996. Mutations in the kinase Rsk-2 associated with Coffin-Lowry syndrome. Nature 384: 567-570.

Tzschentke TM. 1998. Measuring reward with the conditioned place preference paradigm: A comprehensive review of drug effects, recent progress, and new issues. Prog Neurobiol 56: 613-672.

Yang X, Matsuda K, Bialek P, Jacquot S, Masuoka HC, Schinke T, Li L, Brancorsini S, Sassone-Corsi P, Townes TM, et al. 2004. ATF4 is a substrate of RSK2 and an essential regulator of osteoblast biology; implication for Coffin-Lowry Syndrome. Cell 117: 387-398.

Zeniou M, Ding T, Trivier E, Hanauer A. 2002. Expression analysis of $R S K$ gene family members: The RSK2 gene, mutated in Coffin-Lowry syndrome, is prominently expressed in brain structures essential for cognitive function and learning. Hum Mol Genet 11: 2929-2940.

Received March 22, 2011; accepted in revised form June 29, 2011. 


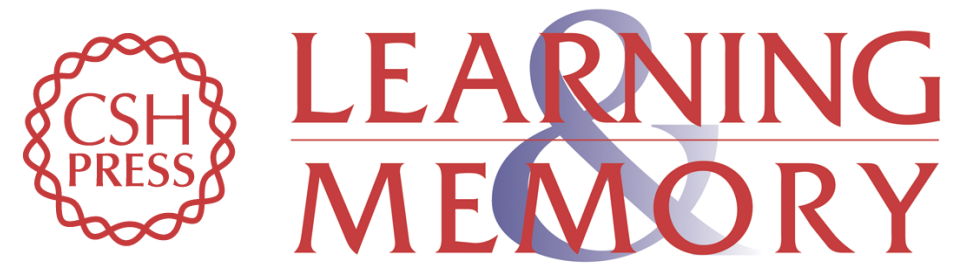

\section{RSK2 signaling in brain habenula contributes to place aversion learning}

Emmanuel Darcq, Pascale Koebel, Carolina Del Boca, et al.

Learn. Mem. 2011, 18:

Access the most recent version at doi:10.1101//m.2221011

\section{Supplemental http://learnmem.cshlp.org/content/suppl/2011/08/18/18.9.574.DC1 Material}

References This article cites 21 articles, 4 of which can be accessed free at: http://learnmem.cshlp.org/content/18/9/574.full.html\#ref-list-1

License

Email Alerting

Receive free email alerts when new articles cite this article - sign up in the box at the Service top right corner of the article or click here. 